Case Report

\title{
Violet LED with low concentration carbamide peroxide for dental bleaching:
}

\section{A case report}

\author{
Alessandra Nara de Souza Rastelli ${ }^{\mathrm{a}, *}$, Hércules Bezerra Dias ${ }^{\mathrm{a}}$, Emanuelle Teixeira Carrera ${ }^{\mathrm{a}}$, \\ Ana Cláudia Pedroso de Barros ${ }^{\mathrm{a}}$, Diego Dantas Lopes dos Santos ${ }^{\mathrm{b}}$, Vitor Hugo Panhóca ${ }^{\mathrm{c}, \mathrm{d}}$, \\ Vanderlei Salvador Bagnato ${ }^{\mathrm{d}}$ \\ a Department of Restorative Dentistry, Araraquara School of Dentistry, University of São Paulo State - UNESP, Araraquara, São Paulo, 14801-903, Brazil \\ ${ }^{b}$ Department of Dental Materials and Prosthodontics, Araraquara School of Dentistry, University of São Paulo State - UNESP, Araraquara, São Paulo, 14801-903, Brazil \\ ${ }^{\mathrm{c}}$ Federal University of São Carlos - UFSCar, São Carlos, São Paulo, Brazil \\ d Physics Institute of São Carlos - IFSC, University of São Paulo - USP, São Carlos, São Paulo, 13566-590, Brazil
}

\section{A R T I C L E I N F O}

\section{Keywords:}

Dental bleaching

Color

Dentin sensitivity

LED

Carbamide peroxide

\begin{abstract}
A B S T R A C T
The use of violet LED was recently introduced as a new alternative technique to perform tooth bleaching, associated or not with bleaching agents. This paper shows a tooth bleaching clinical case report performed in a 26year-old woman, using a violet LED with $10 \%$ carbamide peroxide (CP). The tooth bleaching protocol was performed in 3 sessions. Fifteen irradiations using a violet light system (BMW, MMoptics, São Carlos, SP, Brazil) were done during $30 \mathrm{~s}$ (on) and $60 \mathrm{~s}$ (off) without bleaching gel, and more 5 irradiations associated to $10 \%$ CP. Upper and lower arches were irradiated separately. The total procedure time was $30 \mathrm{~min}$. Subjective and objective color assessments were performed using Vitapan Classical shade guide and VITA Easyshade spectrophotometer, respectively. Dentin sensitivity was measured using a visual analogue scale. The use of violet LED associated to a low concentration carbamide peroxide successfully promoted tooth bleaching and no side effects i.e. dentin sensitivity during and post treatment was observed.
\end{abstract}

\section{Introduction}

One of the main treatments used to obtain a satisfactory and lowcost dental aesthetic is the tooth bleaching performed in-office under high concentrations of hydrogen peroxide $(35 \%-38 \%)$ or at-home/ dentist-prescribed under lower concentrations $(5 \%-22 \%)$ or one of the precursor, carbamide peroxide [1].

LED light source has been used to improve the bleaching process, but the literature is controversy about the efficacy of this application [2]. The LED is applied only as an adjunct in tooth bleaching process, accelerating chemical reactions involved, but not as a bleaching agent by itself [2].

The major side effect is tooth sensitivity. After bleaching, the athome use of toothpaste, mouthwashes, and chewing gums are reported as a simple treatment to reduce the sensitivity [3]. On the other hand, in-office treatments can offer a wide range of options, such as the use of low-level lasers combined or not with topical desensitizing agents [3].
However, a new generation of LED under violet wavelength with the ability to provide tooth bleaching in the absence of hydrogen or carbamide peroxide has recently introduced [4-6]. This innovative equipment uses a technology based on violet LED for generating of the light, which allows performing the bleaching process in absence or in association of bleaching gels, even for patients with dentin hypersensitivity.

Therefore, the purpose of this report was to describe the application of this innovative technology based on violet LED, to evaluate the subjective and objective changes on tooth color of a patient after bleaching associated to $10 \%$ carbamide peroxide.

\section{Case report}

A 26-year-old woman reported to the Department of Restorative Dentistry at Araraquara School of Dentistry, University of São Paulo State - UNESP (São Paulo, Brazil) looking for tooth bleaching treatment

\footnotetext{
* Corresponding author at: Department of Restorative Dentistry, Araraquara School of Dentistry, University of São Paulo State - UNESP, 1680 Humaitá St., MailBox: 331, ZipCode: 14.801-903, Araraquara, São Paulo, 14801-903, Brazil.

E-mail addresses: alrastelli@foar.unesp.br (A.N.d.S. Rastelli), herc_dias@yahoo.com.br (H.B. Dias), manu_teixeira8@hotmail.com (E.T. Carrera), anacpedroso@foar.unesp.br (A.C.P. de Barros), diego-dantas-tm@hotmail.com (D.D.L. dos Santos), vhpanhoca@msn.com (V.H. Panhóca), vander@if.sc.usp.br (V.S. Bagnato).
} 

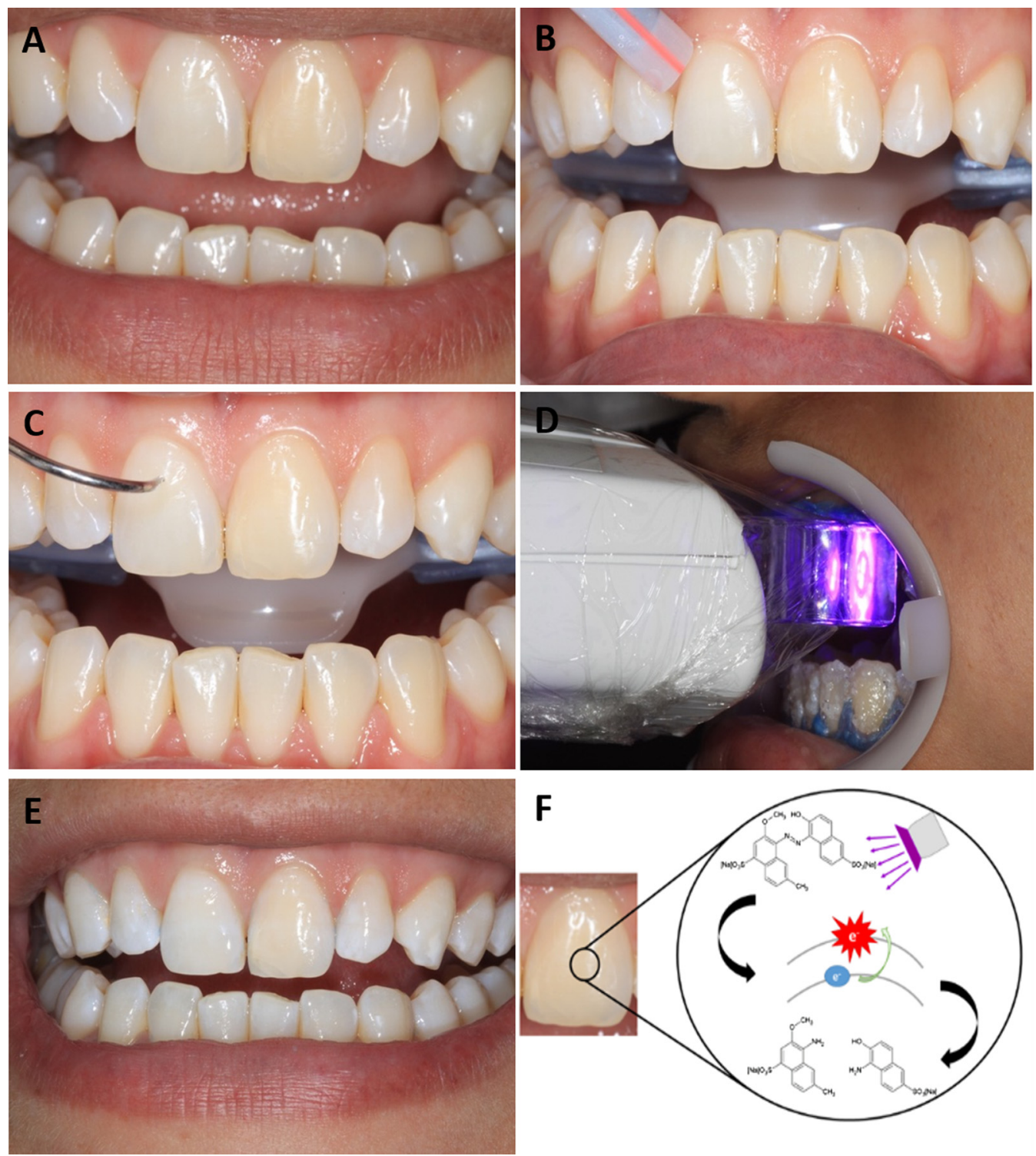

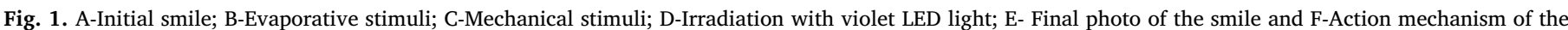
violet LED.

Table 1

Mean $\Delta \mathrm{SGU}$ and $\Delta \mathrm{E}^{*}$ values measured at different bleaching sessions.

\begin{tabular}{|c|c|c|c|c|c|c|c|}
\hline \multicolumn{2}{|l|}{ Protocol and irradiation number } & \multicolumn{3}{|l|}{$\Delta \mathrm{SGU}$} & \multicolumn{3}{|l|}{$\Delta \mathrm{E}^{*}$} \\
\hline & & Session 1 & Session 2 & Session 3 & Session 1 & Session 2 & Session 3 \\
\hline \multirow[t]{3}{*}{ LED irradiation } & 5 & 0,5 & 1,1 & 1,2 & 5,3 & 3,8 & 3,4 \\
\hline & 10 & 0,9 & 1,1 & 1,2 & 5,0 & 4,1 & 3,8 \\
\hline & 15 & 1,1 & 1,1 & 1,2 & 5,7 & 4,1 & 3,9 \\
\hline \multirow[t]{2}{*}{ LED $+10 \%$ carbamide peroxide } & 20 & 1,1 & 1,1 & 1,2 & 5,3 & 4,3 & 3,3 \\
\hline & After polishing & 1,1 & 1,1 & 1,2 & 5,2 & 3,5 & 3,1 \\
\hline
\end{tabular}

because she was unsatisfied with the color of her teeth (Fig. 1A). A composite resin restoration was observed in the left upper central incisor, good periodontal health, absence of gingival retraction and the patient reported no experience previous dental sensitivity.

Shade register was performed using a shade guide Vitapan Classical (Vita Zahnfabrik, H. Rauter GmbH \& Co. KG.D-7880 Säckingen, Germany) and a reflectance spectrophotometer VITA Easyshade Advance (Vident, Brea, CA, USA, Lot: H25543), these qualitative data were transformed into quantitative data in order to calculate the variation of the color scale units ( $\Delta$ SGU - shade guide units) [2].

The Visual Analogue Scale (VAS) was used to determine the tooth sensitivity following application of evaporative and tactile tests (Fig.1BC). The evaluation was performed before bleaching, after 5, 10, 15, 20 irradiations, and after tooth enamel polishing, indicating different scores on the VAS scale. Three sessions were performed (Fig.1E).

Before the application of the light source based on violet LED under $408 \mathrm{~nm}$ (Bright Max MMO - BMW Equipamentos Opto Eletronicos, Sao Carlos, SP, Brazil) during $30 \mathrm{~s}$ (Fig. 1D), a gingival barrier was applied 
(Top Dam, FGM Produtos Odontológicos, Ltda, Joinvile, Santa Catarina, Brazil). The irradiation of $30 \mathrm{~s}$ was repeated 20 times, and before the last 5 irradiations, a translucent $10 \%$ carbamide peroxide (Whiteness Perfect, FGM Produtos Odontológicos, Joinville, Santa Catarina, Brazil) was used.

\section{Results}

The mean values of shade guide units ( $\triangle \mathrm{SGU}$ ) (Table 1 ) showed that the initial 10 irradiations led to an improvement of shade guide units at the first session, which was maintained constant during the second session and was slightly increased at the third session. According to these data, the use of violet LED was enough to promote the bleaching without $\mathrm{CP}$ association. Additionally, the data reveals that polishing procedure did not interfere on the values of shade guide units.

The $\Delta \mathrm{E}^{*}$ mean values (Table 1 ) reveal that spectrophotometer measurements did not differ between the irradiation during the first session. However, during the second session, an appreciable color change was noted (from $\Delta \mathrm{E}^{*}=5.3$ during the first session to $\Delta \mathrm{E}^{*}=3.8$ at the end of the second session). This noticeable color change was maintained until the end of the third session $\left(\Delta \mathrm{E}^{*}=3.1\right)$. On the contrary to $\Delta$ SGU findings, the polishing led to reducing of $\Delta \mathrm{E}^{*}$ mean values, especially in the second session.

The trend analysis of color change based on subjective and objective shows that $\Delta$ SGU means values increased while $\Delta \mathrm{E}^{*}$ means values decreased over the bleaching sessions. The linear trend was observed in the first session for shade guide units and over the sessions for color stability.

\section{Discussion}

The tooth bleaching was successfully achieved, but the restorative material in the upper left central incisor was not bleached (Fig. 1E), and no sensitivity during and after bleaching sessions, even before the association with carbamide peroxide was found.

The use of $35 \%$ hydrogen peroxide has been presented as the first choice for the most of clinicians to perform in-office bleaching, due the ability to dissociation in oxygen reactive species [1]. However, in this current case report, $10 \%$ carbamide peroxide was used in association with violet LED to perform in-office tooth bleaching. The violet light $(408 \mathrm{~nm})$ was used due the ability to promote bleaching and interact with biological molecules without promoting molecular damage [4-6].

Heat-activated therapies and LED light systems are usually used to enhance or accelerate the action of the bleaching agents [1]. The effectiveness of these methods and the side effects has been extensively discussed in several clinical reports, research studies and reviews [1,2].

The use of violet LEDs light sources $(\lambda 405-410 \mathrm{~nm})$ to promote tooth bleaching without using bleaching gels was found to be a new alternative to perform in-office bleaching [4-6].

The use of violet LED has been presented in the clinical scenario as a good alternative to perform tooth bleaching without generating dental pulp heat and dental sensitivity. The use of this light system associated with low concentrations of carbamide peroxide was found to be a good option to fulfill the rising demand for aesthetics, promoting efficient tooth bleaching without side effects during and post treatment.

\section{Acknowledgments}

The authors thank the São Paulo Research Foundation (FAPESP Process Number 2013/ 07276-1 and 2017/17839-4) for the financial support for this study.

\section{References}

[1] W. Buchalla, T. Attin, External bleaching therapy with activation by heat, light or laser-a systematic review, Dent. Mater. 23 (2007) 586-596, https://doi.org/10. 1016/j.dental.2006.03.018.

[2] K. Luk, L. Tam, M. Hubert, Effect of light energy on peroxide tooth bleaching, J. Am. Dent. Assoc. 135 (2004), https://doi.org/10.1017/CBO9781107415324.004 194201-229.

[3] M. Asnaashari, M. Moeini, Effectiveness of lasers in the treatment of dentin hypersensitivity, J. Lasers Med. Sci. 4 (2013) 1-7.

[4] A.D.N. Lago, W.D.R. Ferreira, G.S. Furtado, Dental bleaching with the use of violet light only: reality or future? Photodiagn. Photodyn. Ther. 17 (2017) 124-126, https://doi.org/10.1016/j.pdpdt.2016.11.014.

[5] V.H. Panhoca, B.P. de Oliveira, A.N.S. Rastelli, V.S. Bagnato, Dental bleaching using violet light alone: clinical case report, Dentistry 7 (2017) 1-4, https://doi.org/10. 4172/2161-1122.1000459.

[6] F. Zanin, Recent advances in dental bleaching with laser and LEDs, Photomed. Laser Surg. 34 (2016) 135-136, https://doi.org/10.1089/pho.2016.4111. 